\section{A solitary gastric metastasis from pulmonary adenocarcinoma: a case report}

A 77-year-old male complained of persistent cough and sputum for 2 months. Chest radiography showed a solitary mass with solid opacity in the right upper lung (RUL) (figure 1A, arrow). A CT scan of the chest demonstrated an irregular mass measuring $3.8 \times 2.8 \mathrm{~cm}$ in the RUL (figure 1B, arrow). Histologically, the tumour was interpreted as a poorly differentiated adenocarcinoma (figure $1 \mathrm{C}-\mathrm{E}$ ).

During the staging investigation, integrated positron emission tomography using $\left[{ }^{18} \mathrm{~F}\right]$ fluoro-2-deoxy-D-glucose (FDG-PET)/CT scan showed intrahepatic biliary dilatations, but it showed no abnormal FDG uptake, except the lung mass on the RUL, in any other organs including the gastrointestinal tract (figure 2A). Hence, we decided to perform endoscopic retrograde cholangiopancreatography (ERCP) to evaluate the biliary dilatations, and the results conclusively ensured that the dilatations were medically insignificant. Routine gastroduodenoscopic examination, prior to ERCP, revealed a single oval-shaped ulcer with a raised margin at the mid-antrum of the stomach (figure $2 \mathrm{~B}$ ). Histopathological findings were similar to gastric adenocarcinoma (figure $2 \mathrm{C}$ ). In the immunohistochemical studies, tumour cells were positive for thyroid transcription factor 1 (TTF-1) and cytokeratin 7 (CK-7), which are used for identifying pulmonary origin (figure $2 \mathrm{D}-\mathrm{E}$ ). These findings indicated that the gastric ulcer lesion is a solitary metastasis from a pulmonary adenocarcinoma. The patient underwent right upper lobectomy and subtotal gastrectomy without complications.

Gastric metastasis from lung cancer is not common; its incidence has been reported to be from $0.4 \%$ to $5.1 \%$. A case of gastric metastasis from adenocarcinoma of the lung is very rare. ${ }^{1}$ False-negative results from integrated PET/CT can occur in tumours with low metabolism, such as carcinoid tumours and bronchioloalveolar cell carcinoma. ${ }^{2}$

\section{Min Hee Lee, ${ }^{1}$ So Ri Kim, ${ }^{1}$ Jae Seung Soh, ${ }^{1}$ Myoung Ja Chung, Yong Chul Lee ${ }^{1}$}

${ }^{1}$ Department of Internal Medicine and Research Center for Pulmonary Disorders, Chonbuk National University Medical School, Jeonju, South Korea; ${ }^{2}$ Department of Pathology, Chonbuk National University Medical School, Jeonju, South Korea

Correspondence to Yong Chul Lee, Department of Internal Medicine, Chonbuk National University Medical School, San 2-20 Geumam-dong, Deokjin-gu, Jeonju, Jeonbuk 561-180, South Korea; leeyc@chonbuk.ac.kr

Acknowledgements We thank Professor Mie-Jae Im for critical readings of the manuscript.
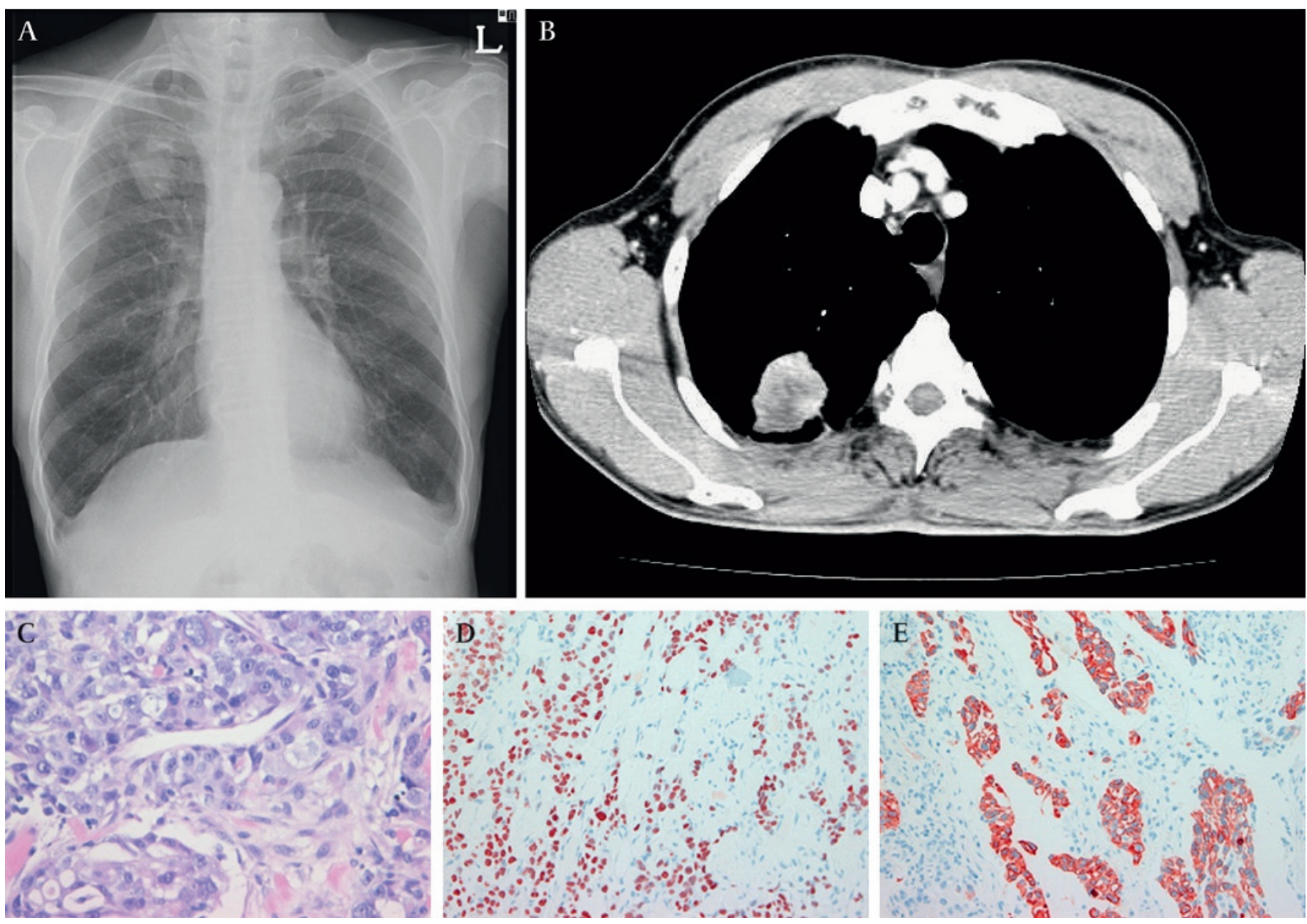

Figure 1 (A) Chest $x$-ray shows a round mass in the right upper lobe measuring $\sim 4 \mathrm{~cm}$. (B) CT scan of the chest demonstrates a solitary round mass in the right upper lobe measuring $3.8 \times 2.8 \mathrm{~cm}$ with a speculated margin, pleural tagging and focal enhancement. (C) H\&E staining of the lung mass tissues. This shows a papillary pattern and undifferentiated diffuse proliferation with minimal glandular formation, and neoplastic cells including a large nucleus with prominent nucleoli, findings compatible with poorly differentiated adenocarcinoma. The positivity of (D) thyroid transcription factor 1 (TTF-1) and (E) cytokeratin 7 (CK-7) stained in brown was determined by immunohistochemistry. 

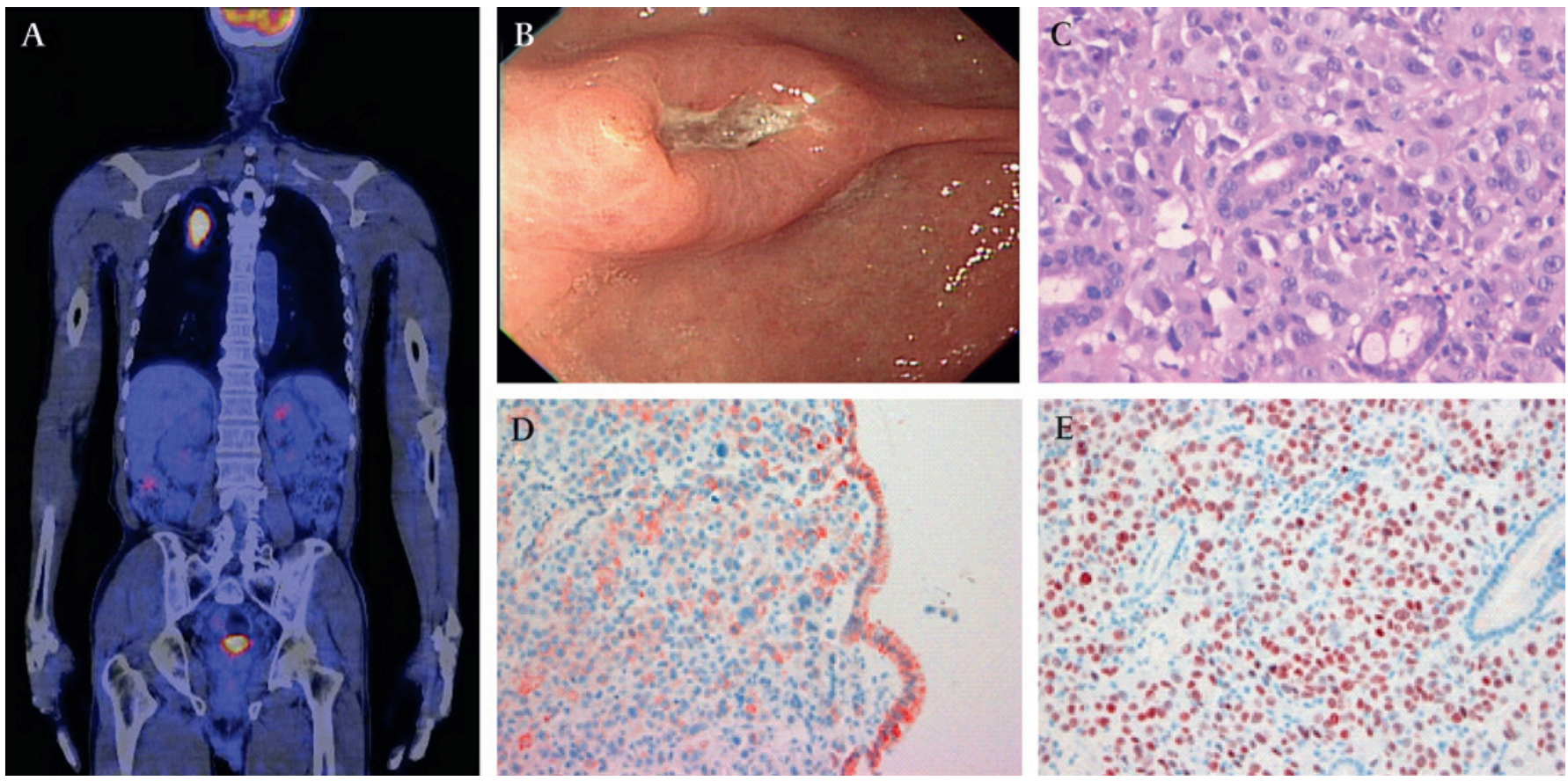

Figure 2 (A) An integrated positron emission tomography (PET)/CT scan shows no abnormal $\left[{ }^{18} \mathrm{~F}\right]$ fluoro-2-deoxy-D-glucose (FDG) uptake in the gastrointestinal tract. (B) A single large oval-shaped ulcer with a raised margin of $2 \times 1.5 \mathrm{~cm}$ at the mid-antrum in the stomach. (C) H\&E staining in the gastric ulcer tissues. This shows a papillary pattern and cohesive neoplastic cells forming irregular gland-like tubular structures, findings compatible with poorly differentiated adenocarcinoma. The positivity of (D) thyroid transcription factor 1 (TTF-1) and (E) cytokeratin 7 (CK-7) stained in brown was determined by immunohistochemistry.

\section{Learning points}

Doctors should be aware of the possibility of gastrointestinal tract metastases even though the patient with primary lung cancer has no specific gastrointestinal symptoms.

- Immunohistochemical study is very helpful for the identification of the exact origin of a metastatic tumour when biopsy results are ambiguous by histology alone.

Funding This study was supported by a grant of the Korea Healthcare technology R\&D Project, Ministry for Health, Welfare and Family Affairs, Republic of Korea (A084144) and by the Korea Science and Engineering Foundation (KOSEF) through the National
Research Lab. Program funded by the Ministry of Education, Science and Technology (ROA-2005-000-10052-0(2008)).

\section{Competing interests None.}

Patient consent Obtained.

Provenance and peer review Not commissioned; externally peer reviewed.

Accepted 22 September 2009

Thorax 2010;65:661-662. doi:10.1136/thx.2009.122382

\section{REFERENCES}

1. Yoshimoto A, Kasahara K, Kawashima A. Gastrointestinal metastases from primary lung cancer. Eur J Cancer 2006;42:3157-60.

2. Antoch G, Vogt FM, Freudenberg LS, et al. Whole-body dual-modality PET/CT and whole-body MRI for tumor staging in oncology. JAMA 2003;290:3199-206. 